Musées, Patrimoine et Culture scientifiques et techniques

$111 \mid 2007$

mai - juin 2007

\title{
Gratuité des musées et valeur perçue par les publics
}

Dominique Bourgeon-Renault, Anne Gombault, Marine Le Gall-Ely, Christine Petr et Caroline Urbain

\section{(QpenEdition Journals}

Édition électronique

URL : http://journals.openedition.org/ocim/764

DOI : 10.4000/ocim.764

ISSN : 2108-646X

Éditeur

OCIM

Édition imprimée

Date de publication : 1 mai 2007

Pagination : $31-39$

ISSN : 0994-1908

Référence électronique

Dominique Bourgeon-Renault, Anne Gombault, Marine Le Gall-Ely, Christine Petr et Caroline Urbain, «Gratuité des musées et valeur perçue par les publics », La Lettre de I'OCIM [En ligne], 111 | 2007, mis en ligne le 07 septembre 2011, consulté le 01 mai 2019. URL : http://journals.openedition.org/ ocim/764; DOI : 10.4000/ocim.764 


\title{
Gratuité des musées et valeur perçue par les publics
}

\author{
Dominique Bourgeon-Renault, Anne Gombault, Marine Le Gall-Ely, \\ Christine Petr et Caroline Urbain *
}

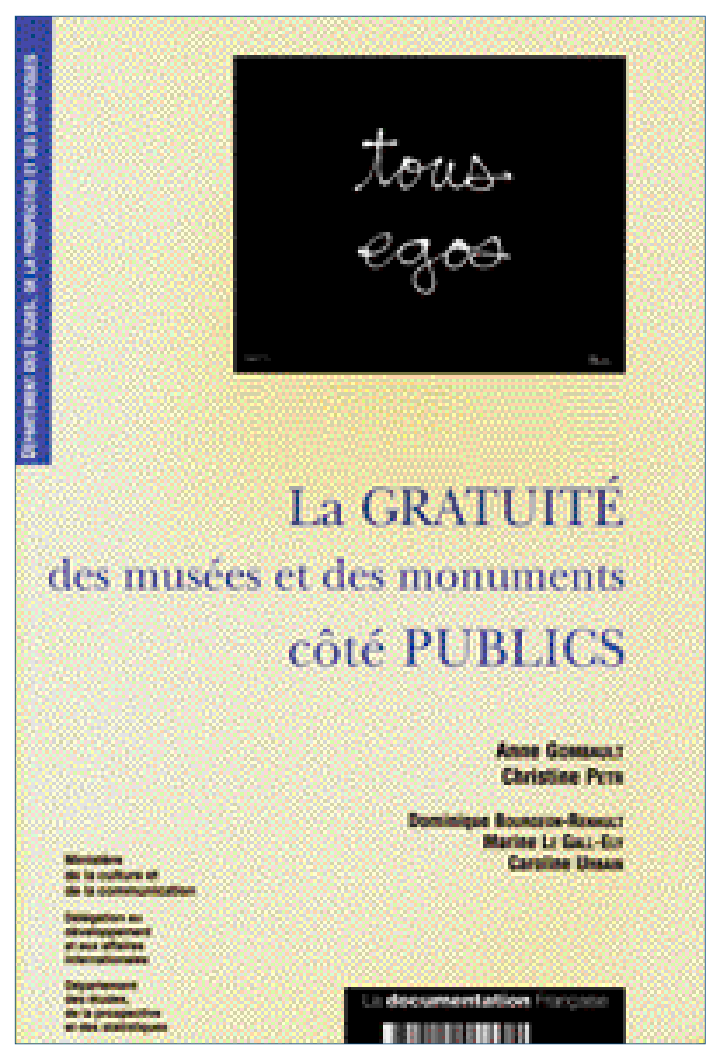

* Dominique Bourgeon-Renault est professeur à l'université de Bourgogne, membre du laboratoire CIMEOS et membre associé à la chaire Arts, Culture et Management en Europe (CEREBEM) Anne Gombault est professeur titulaire de la chaire Arts, Culture et Management en Europe (CEREBEM), Bordeaux, École de Management

Marine Le Gall-Ely, est maître de conférences à l'université de Rennes 2, ICI EA2652

Christine Petr est maître de conférences IGR à l'université de Rennes 1, CREM UMR CNRS 6211

Caroline Urbain est maître de conférences à l'université de Nantes, CRGNA
Analyser les comportements des français face à la gratuité et en particulier étudier la relation existant entre cette gratuité et d'une part la représentation que le public se fait des musées et d'autre part la valeur qu'il attribue dans ces conditions à son expérience de visite : telles sont les principaux éléments de réflexion développés ici par les auteurs.

\section{Introduction}

Valeur fondatrice des musées, sceau de leur identité originelle, la gratuité avait progressivement disparu au XXe siècle en France pour laisser la place à des politiques tarifaires de plus en plus sophistiquées. Depuis les années 1990, elle fait un retour marquant. Le mouvement a débuté au Royaume-Uni et s'est poursuivi dans toute l'Europe (Gombault, 2002). En France, il débute en 1996. Sur proposition du ministère de la Culture et de la Communication, le musée du Louvre devient gratuit le premier dimanche de chaque mois, à titre expérimental pour deux ans : c'est un succès. Aussi en 1998, la mesure est-elle reconduite sans limitation de durée. En 2000, elle est mise en œuvre dans l'ensemble des musées nationaux et des monuments historiques. Puis, plusieurs villes l'adoptent dans les musées municipaux : en 2002, les musées municipaux parisiens offrent une gratuité permanente, bientôt rejoints par ceux de Dijon, Caen, Bordeaux ou encore du département de l'Isère. Cette évolution, qui peut sembler paradoxale dans un monde muséal pragmatique intégrant de plus en plus l'appel au marché, marque en fait l'apparition de stratégies 
de prix où la gratuité, une action-prix comme une autre, sert les objectifs d'accessibilité et de promotion de ces institutions (Gombault, 2005).

Qu'en pensent les publics ? De nombreuses études quantitatives d'impact sur la fréquentation ont été menées principalement à l'étranger (Bailey et al., 1997). Elles décrivent toutes une augmentation de la fréquentation à court terme, qualifiée d'« effet lune de miel »(Dickenson, 1993), mais montrent qu'à plus ou moins long terme, la mesure de gratuité en tant que telle n'aurait pas d'effet sur la fréquentation. Seule une politique d'accompagnement actif de cette mesure, visant à en faire un outil d'implication dans la visite pour les publics, peut produire des résultats. Pourtant, au-delà de ces études d'impact, rares sont les travaux à s'être intéressés à la réception de la mesure de gratuité, et au sens donné par les publics à la gratuité et à leur expérience vécue lors de la visite. En France, on recense seulement l'étude de Gottesdiener et Godrèche (1996), qui s’intéresse aux représentations des dimanches gratuits des publics du musée du Louvre. Pour combler ce manque, le département des Études, de la Prospective et des Statistiques du ministère de la Culture et de la Communication, après avoir mené une série d'études quantitatives sur les effets des mesures de gratuité dans les musées et les monuments nationaux, a confié à une équipe de cinq enseignants chercheurs, en sciences de gestion, la réalisation d'une étude principalement qualitative (Gombault, Petr, BourgeonRenault, Le Gall et Urbain, 2006). La recherche visait à comprendre les représentations, les projets d'usage et les comportements des Français face à la gratuité des musées et monuments. Après avoir présenté cette étude et résumé ses principaux résultats, cet article développe l'un d'entre eux : l'importance de

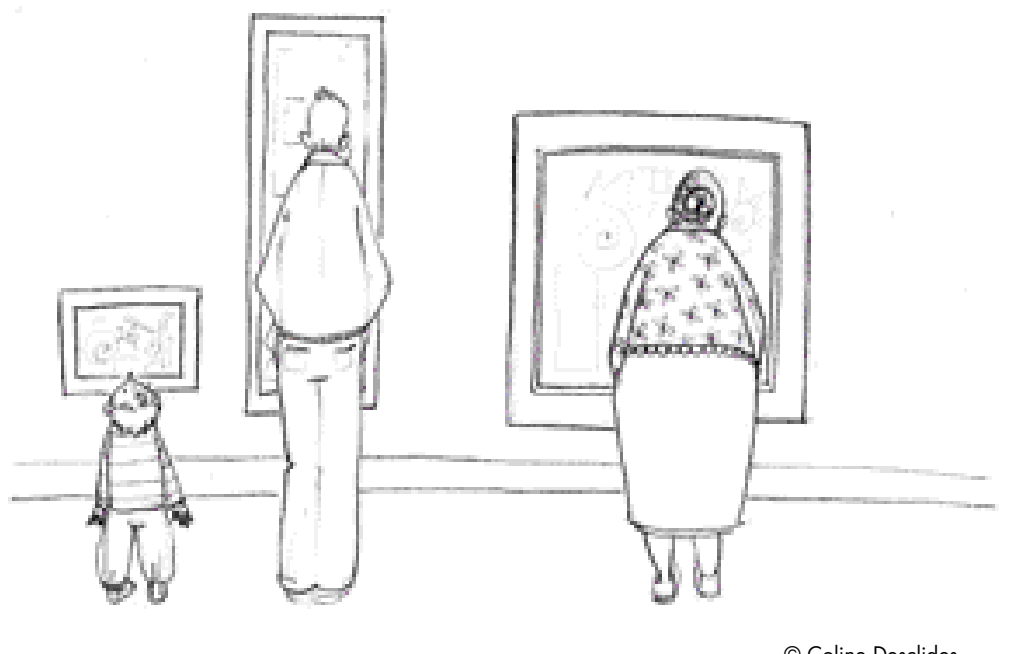

(c) Coline Desclides la valeur pour les publics dans un contexte de gratuité. Quelle valeur attachent-ils à la visite d'un musée dont l'entrée est gratuite ? Existe-t-il une relation entre ces représentations de la gratuité et la valeur attachée aux musées et à leur visite ?

\section{La gratuité des musées côté publics : présentation de la recherche}

\section{Problématique et méthodologie}

La problématique générale de la recherche commandée par le département des Études, de la Prospective et des Statistiques a été formulée de la façon suivante : dans le contexte français, quelles représentations de la gratuité des musées et des monuments, les publics ont-ils et comment celles-ci sont-elles reliées à leurs représentations, projets d'usage et comportements de fréquentation des musées et des monuments?

La contribution attendue était de générer une connaissance théorique sur les représentations, les projets d'usage et les comportements des publics, face à la gratuité des musées et des monuments, à partir de données empiriques, et par un raisonnement inductif et itératif, mobilisant des théories pertinentes. Cet objectif exploratoire a été servi par une stratégie de recherche dite de «multiangulation » consistant à comparer de multiples sources de données empiriques et théoriques pour produire de la connaissance. Toutes les tactiques de «multiangulation » ont été utilisées afin de garantir une fiabilité et une validité élevée : «multiangulation » des données, des techniques de production des données, des interprétations produites par les personnes étudiées, des théories, des chercheurs et enfin des paradigmes. La production des données s'est organisée de la façon suivante : un mode central de production des données - 52 entretiens individuels - complété par trois modes complémentaires : 4 entretiens de groupe, 36 observations et entretiens individuels sur site et une enquête quantitative - 580 questionnaires. Le critère central d'échantillonnage de chaque phase de production a été la fréquence de visite : visiteurs habitués (plus de 2 visites dans l'année écoulée), visiteurs occasionnels (au moins une visite dans les cinq dernières années et au plus deux visites dans l'année écoulée), non-visiteurs (qui n'ont pas effectué de visite depuis cinq ans.).

Les données ont été collectées en 2002 et 2003, de façon synchronique : partout en France, auprès de français (les touristes étrangers n'ont pas été étudiés), en distinguant entre la région Parisienne et les autres régions et entre les zones urbaines et les zones rurales 

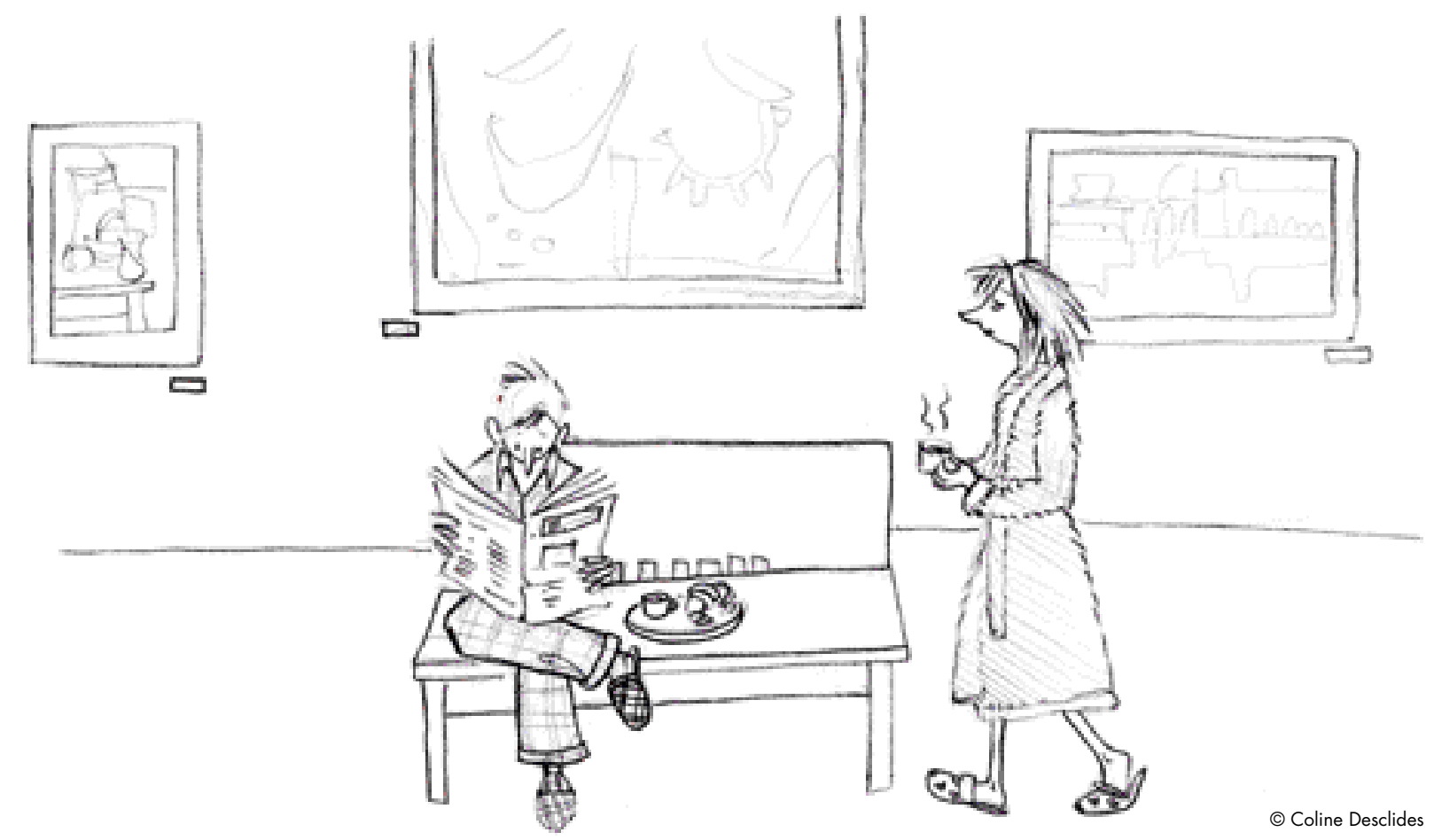

pour les entretiens et l'enquête ; et dans deux sites-tests en Bourgogne - un musée (Magnin à Dijon) et un monument (Châteauneuf-en-Auxois) - en comparant des configurations tarifaires différentes (dimanche payant, dimanche gratuit, Journées du Patrimoine) pour les observations et entretiens de visite. Des analyses de contenu thématiques, empiriques puis théoriques ont été menées par phase et globalement. Elles ont permis de formuler 27 propositions théoriques à valeur d'hypothèses, qui ont été ensuite reprises, discutées et réduites à trois méta-propositions (agrégats de propositions, visant à un niveau de connaissance théorique synthétique et général sur la question abordée).

\section{Trois grands résultats}

Le premier résultat de l'étude montre, que pour les publics, à l'encontre d'une idée reçue, la gratuité est secondaire dans la construction et la réalisation d'un projet de visite. Elle est loin d'être la clé d'entrée de la fréquentation des musées et des monuments, qui réside avant tout dans l'implication des individus dans cette activité. Pour autant, la gratuité n'est pas neutre et dispose d'un pouvoir métamorphique sur la pratique de visite de musées et de monuments, qui s'exerce de trois façons différentes :

- elle permet de faire penser aux musées et aux monuments ;

- elle améliore l'accessibilité objective et symbolique de l'offre des musées et des monuments ;

- elle change le processus de décision de visite qui apparaît plus simple, moins coûteux, et basé alors sur l'essai et l'exploration expérientielle.
Le second résultat de l'étude montre que les représentations des musées et des monuments s'inscrivent globalement dans un cadre de référence unique, de biens communs culturels dans un système marchand, que la gratuité interroge. Dans une logique collective, la gratuité des musées et des monuments est perçue en France de manière équivoque : ambivalente (envisagée différemment pour le collectif ou pour soi), paradoxale dans ses effets, ambiguë dans son contenu même. Ce caractère équivoque peut être interprété comme caractéristique des représentations de la gratuité des biens sociaux en général dans une société marchande, et donc non spécifique aux musées et aux monuments. Par suite, dans les représentations des publics, la politique de gratuité des musées et des monuments en France apparaît de façon unanime comme étant mal adaptée. Le manque de cohérence et une communication déficiente sont mis en cause. Au-delà de ces deux points de consensus, les représentations de la politique de gratuité des musées et des monuments divergent fortement quant à sa légitimité, ses objectifs et son financement.

Dans une logique individuelle, la gratuité ramène les publics à la signification du fait de payer. Le fait de payer est perçu comme un visa d'entrée dans les musées et les monuments, comme un engagement dans l'acte de visite, même s'il crée une distance entre les musées et les monuments et leurs publics. Dans les représentations des publics, la gratuité est fortement associée à la valeur des musées et des monuments et de l'expérience de visite, qui est 
considérée comme inscrite dans une relation d'échange. Ensuite, selon que les publics se déclarent intéressés ou non par les musées et les monuments, selon les valeurs qui les animent, selon les attentes vis-à-vis de la pratique, et selon la pratique elle-même, les représentations de la gratuité sont très différentes. Ainsi, quatre univers fragmentent le cadre de référence et sont identifiés ainsi :

- un univers sacré, marqué par une vision dogmatique et esthétique et dans lequel la gratuité, considérée comme naturelle, est un principe qui fait débat ;

- un univers patrimonial au sens culturel, mais également au sens économique et juridique, dans lequel la gratuité, très controversée, apparaît comme un idéal impossible ;

- un univers de loisirs offrant des prestations de services dans une logique de consommation, dans laquelle la gratuité est considérée comme une opportunité ;

- un univers étrange, dans lequel les musées et les monuments sont tellement absents que la gratuité ne fait pas sens.

Intégrant des considérations de marché, les univers patrimonial et de loisirs, auxquels appartiennent des visiteurs habitués, occasionnels et des non-visiteurs, se distinguent nettement de l'univers sacré, porté principalement par des visiteurs initiés aux musées et aux monuments.

Enfin, le troisième résultat de l'étude montre que pour certains visiteurs et à certaines conditions, l'expérience de la visite gratuite des musées et des monuments peut permettre un apprentissage de leur visite ainsi que l'appropriation de la mesure de gratuité. D'abord, l'apprentissage de la pratique serait facilité par les effets de la gratuité sur l'image de la pratique et des objets de visite. En effet, la gratuité modifie la perception des musées et des monuments et le projet d'usage de ces lieux. Elle change le processus de décision : la décision d'une visite gratuite exige moins d'efforts et de calculs, permet de s'appuyer sur l'essai au lieu de l'évaluation, et supprime l'acte d'achat matérialisé par le paiement. Ensuite, l'expérience d'une visite gratuite semble donner plus souvent lieu à de nouveaux projets de visites gratuites, voire leur possible choix exclusif. Ainsi, l'expérience gratuite favoriserait l'appropriation individuelle des mesures de gratuité. Enfin, lors de son vécu, la gratuité fait vivre une expérience de visite différente de celle de la configuration payante :

- elle modifie l'usage des lieux ; les comportements changent et deviennent plus détendus, voire profanes, avec des variations selon le type de lieu ou selon le type de gratuité ;

- elle crée un contexte de convivialité spécifique et modifie le lien social inter-groupe et intra-groupe (expérience collective partagée).

Les représentations négatives des conditions de visite, dans un contexte de gratuité, ne sont alors pas toujours confirmées lors de l'expérience de visite qui est plutôt vécue de manière positive, d'autant plus que la gratuité événementielle permet des « retrouvailles communautaires » autour de l'objet patrimonial. Ainsi, dans le cadre exceptionnel des visites des Journées du Patrimoine, la gratuité est l'occasion de vivre une expérience partagée autour d'un même objet. Dans cette situation, les personnes se retrouvent et s'accordent autour du patrimoine, au-delà de leurs individualités.

\section{La place de la question de la valeur} dans les résultats

Une partie des résultats de l'étude montre donc l'importance dans les représentations des publics, quand la gratuité est évoquée, de la question de la valeur attachée aux musées et monuments et à l'expérience de visite, et son incidence sur la décision de visite et les pratiques.

Lidentification d'univers de représentations des musées et des monuments montre qu'une majorité de personnes les pensent dans un cadre de référence de l'échange, comme des biens communs culturels dans un système marchand. Ce cadre de référence permet de comprendre les raisons pour lesquelles :
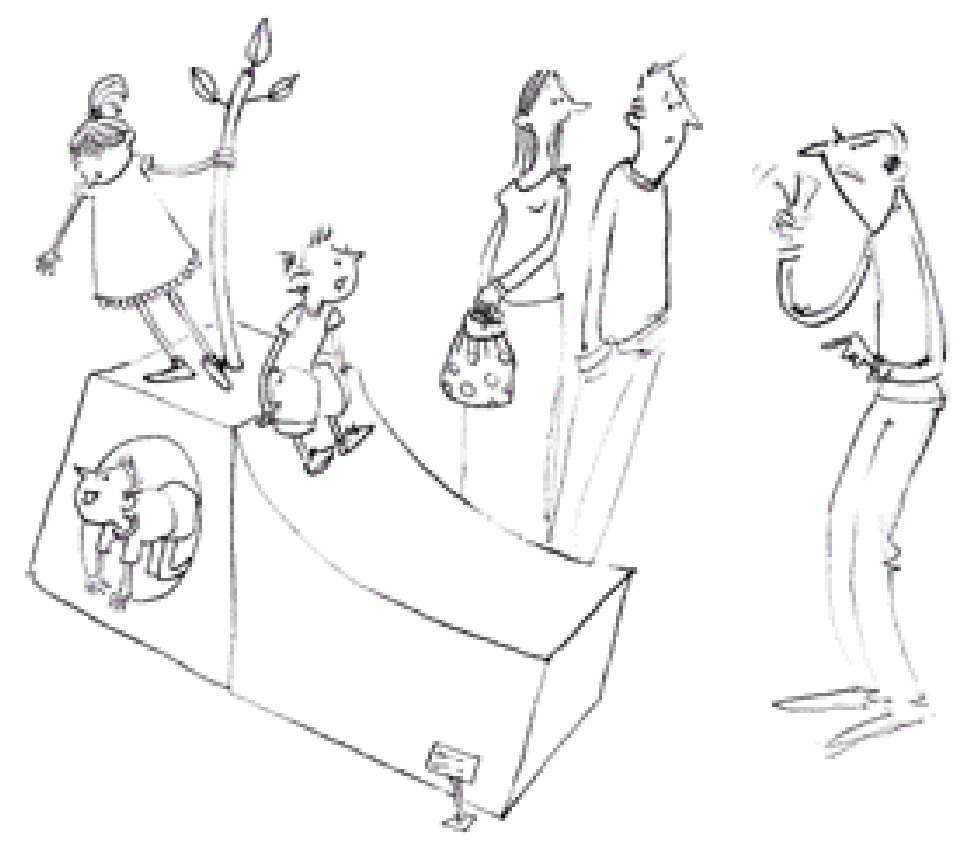

() Coline Desclides 
- dans les représentations des publics, la gratuité se manifeste alors comme un prix, signe majeur d'un monde marchand ;

- le fait de payer est si important pour le visiteur ;

- la gratuité interpelle fortement la valeur des musées et des monuments et de l'expérience de visite.

De plus, la valeur initiale attachée à l'objet culturel reste au centre de la prise de décision de la pratique culturelle qu'il y ait, ou non, proposition de la gratuité. Et quand la gratuité est proposée, les publics envisagent son effet sur la valeur perçue de la pratique. Alors que le premier résultat de notre étude relatif à l'effet secondaire de la gratuité sur la décision de visite et tous les développements qui l'accompagnent sur les raisons de cet effet incitatif mineur, pourraient laisser croire qu'il est possible de clore le débat sur la question de la gratuité dans la culture, en définitive il n'en est rien. La gratuité ne fait pas venir celui qui n'avait pas d'intention initiale d'aller visiter un lieu. Elle ne suffit pas à donner l'envie de visiter à celui qui n'est pas intéressé par l'objet culturel. Elle n'a donc pas le pouvoir prescripteur que ses prosélytes lui prêtent. Cependant elle dispose d'un pouvoir métamorphique qui conduit certains publics à envisager les musées et leur offre de visite, de manière différente. Ils prennent position sur la gratuité, en vertu des qualités ou défauts qu'ils lui attribuent en tant qu'outil modifiant la valeur perçue des musées et de la pratique de visite. Et, en fonction de la valeur qu'ils leur attachent initialement, ils évoquent de manière contrastée, la contribution de la gratuité à cette valeur.

\section{En quoi la gratuité interpelle-t-elle la valeur attachée par les publics aux musées et à leur expérience de visite ?}

Ce questionnement repose sur les représentations de la valeur attachée par les publics aux musées et à leur expérience de visite. Le lien entre, d'une part, le fait de payer ou non l'entrée (valeur d'échange) et, d'autre part, la valeur attachée à la visite d'un musée (valeur d'usage ou expérientielle) est très controversé : la gratuité peut avoir un impact négatif, positif ou neutre sur la valeur perçue de la visite.

La valeur attachée par les publics aux musées et à leur expérience de visite

Les perceptions de la valeur des musées et de l'expérience de visite renvoient aux types de valeur de consommation suivants (Sheth, Newman et Gross, 1991) :
- une valeur fonctionnelle ou capacité des musées à proposer une offre de qualité caractérisée par les thèmes abordés, les œuvres exposées, les lieux et les services mis à la disposition des publics ;

- une valeur sociale liée à la visite pratiquée avec un ou plusieurs groupes sociaux (famille, amis...) ;

- une valeur émotionnelle ou capacité des musées à susciter des émotions, la sensibilité au « beau » ou à l'esthétisme, un sentiment de découverte, d'évasion, de surprise, d'étonnement, de dépassement de soi et d'émerveillement, le développement de l'imaginaire, la nostalgie, une impression de liberté, le plaisir d'être dans les lieux, le désir de nouveauté et de variété, la perception mystique du lieu, la spiritualité... ;

- une valeur épistémique, mettant l'accent sur la capacité des musées à susciter la curiosité et à stimuler le désir de connaissance ;

- une valeur conditionnelle, s'analysant comme le résultat d'une situation spécifique ou d'un ensemble de circonstances (présence de monde ou non, entrée payante ou gratuite, présence d'un guide, occasion particulière de visite...).

Les représentations des musées et de leur valeur sont donc plurielles.

La valeur de l'offre, proposée par les musées et perçue par leurs publics, peut aussi être regardée selon deux perspectives, globale ou analytique (Aurier, Evrard, N'Goala, 2004). La première perspective, qui s'inscrit dans l'échange, appréhende la perception de la valeur globale de l'offre et résulte de la confrontation entre les bénéfices et les sacrifices perçus associés à la consommation (Zeithaml, 1988)

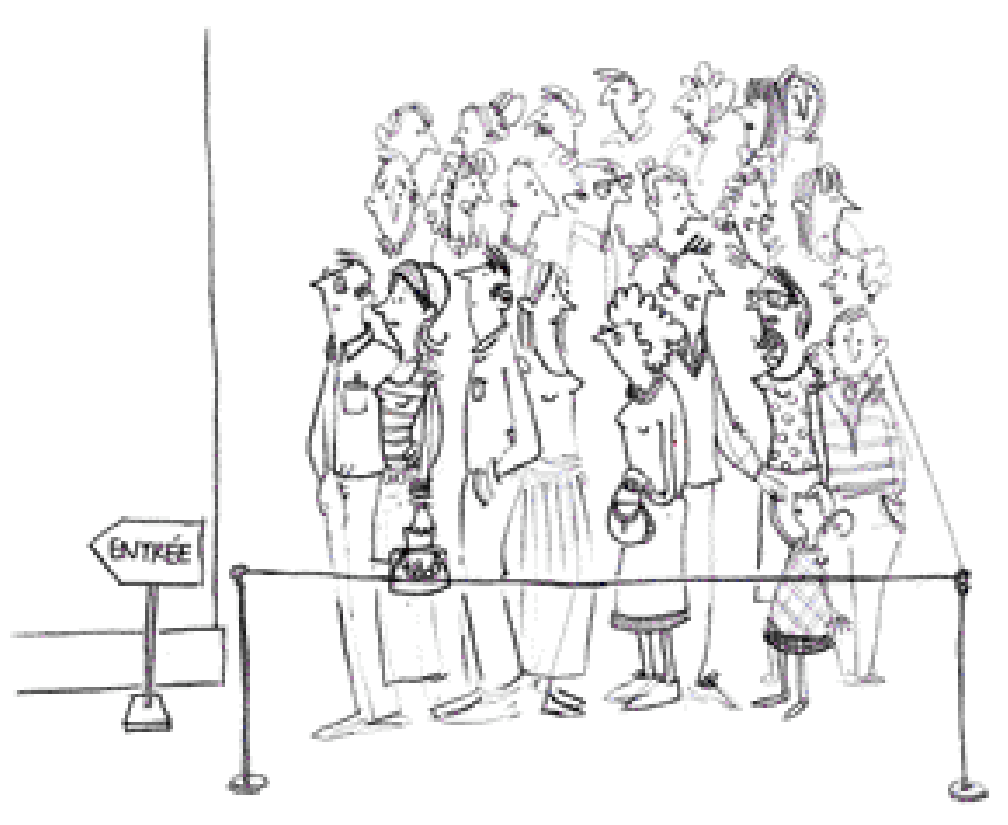

(c) Coline Desclides 
qu'ils soient économiques, psychologiques ou sociaux. Le prix de l'entrée, versus sa gratuité, fait donc partie intégrante de la perception de cette valeur. Il participe à l'ambivalence de la relation gratuité-valeur en pouvant être perçu comme un signe de qualité et comme un effort monétaire pondéré par les efforts non monétaires quiimplique la visite.

La seconde perspective se focalise sur la valeur de la consommation elle-même, c'est-à-dire dans notre contexte, sur le vécu de l'expérience de visite (Holbrook et Hirschman, 1982). Cette approche se situe dans le champ des expériences de consommation ou d'appropriation du lieu. Il s'agit d'une « consommation culturelle » caractérisée par un état subjectif primaire du visiteur provoqué par le symbolisme, l'hédonisme (se fondant sur la capacité d'une exposition à produire du plaisir, de la distraction, des émotions et des sensations) et l'esthétisme (associant la consommation d'œuvres à la beauté) des objets culturels. Cette perspective expérientielle (Bourgeon, 2005) montre que les bénéfices perçus ne reposent pas uniquement sur les aspects matériels, utilitaires et cognitifs des œuvres d'art ou du lieu, mais comprennent des dimensions symboliques, hédonistes et esthétiques, faisant appel à la subjectivité du visiteur et exigeant la prise en compte de sa dimension affective.

La valeur des musées peut ainsi être perçue par les publics sous différentes facettes : valeur symbolique collective, valeur d'échange (valeur dans une perspective transactionnelle), valeur d'usage et/ou d'expérience (valeur de consommation). L'ensemble de ces valeurs participe à la décision de fréquenter un musée. Il s’agit donc de repérer comment les représentations de la gratuité à l'entrée sont susceptibles d'interagir sur la perception de la valeur des musées et de leur visite.

Certains visiteurs considèrent que la gratuité de l'entrée dévalorise ou risque de dévaloriser les musées et/ou l'expérience de visite en diminuant leur statut ou en altérant les conditions de visite. D'autres voient dans la gratuité un moyen de valoriser les musées et l'expérience de visite en ne les réduisant pas à une simple dimension marchande et en permettant de partager leur valeur. Enfin, pour certains, la gratuité ne joue pas sur la valeur qu'ils attribuent aux musées et à leur expérience de visite : en effet, que la visite soit gratuite ou non, certains affirment qu'ils ont tout de même l'intention de visiter les musées.

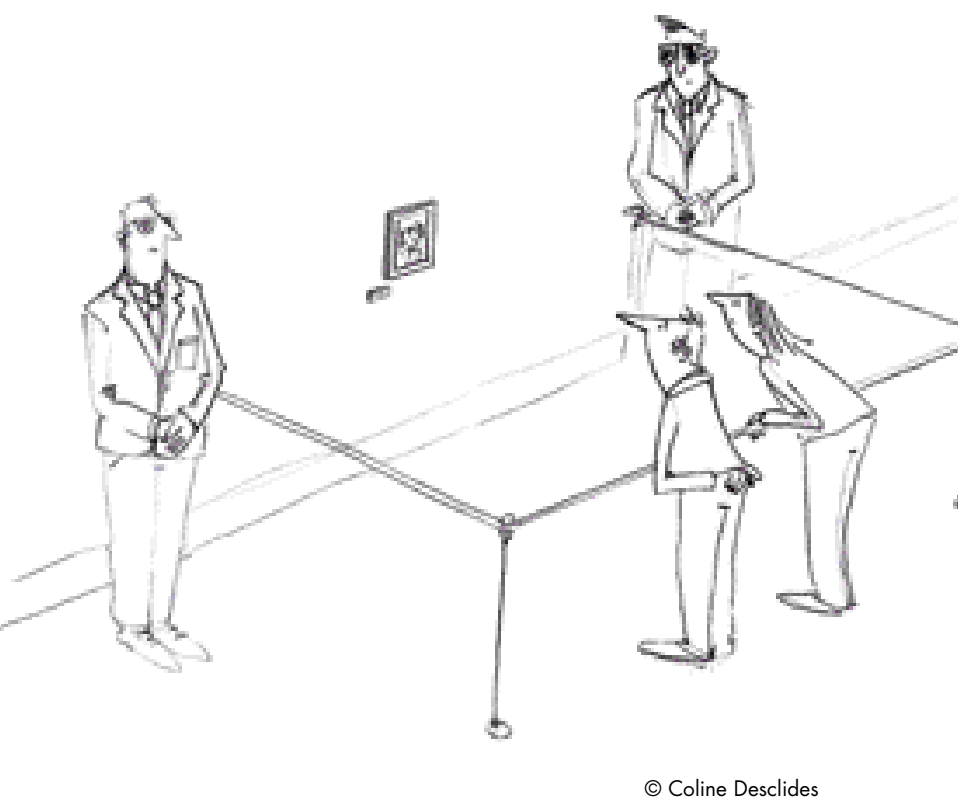

La gratuité : une dévalorisation de l'offre

Les personnes qui voient dans la gratuité un risque de dévalorisation évoquent ainsi les effets symboliques et matériels de la gratuité.

Au niveau symbolique, la gratuité modifie le statut et l’image du lieu : elle le désacralise et, pour certaines personnes, elle risque aussi de banaliser la visite d'un musée, notamment parce qu'elle s'adresse à tous. Elle "galvaude " $(1)$ alors, c'est " le passage au vulgaire, au sens propre, au sens péjoratif du terme ». Plus largement, la gratuité introduit un doute sur la valeur de l'offre : " ce qui est gratuit, ça n'a peut-être pas de valeur [...] cela dévalorise la culture [...] ce qui est beau doit être payé ».

Au niveau pratique, la gratuité est associée à une affluence excessive dans les sites ainsi qu'à une population différente de celle des jours payants, qui peuvent nuire à la qualité de l'expérience de visite : files d'attentes, bruit, manque d'espace... La gratuité est également associée à une moindre qualité de l'offre de services qui est proposée : absence d'accueil et d'information, entretien des équipements, dégradation des lieux.... Les contraintes de planification de la visite (quand la gratuité est périodique) apparaissent aussi plus importantes.

Dans ce cadre, la diminution des bénéfices perçus de l'offre, imputée à la gratuité, prend alors le pas sur la suppression du sacrifice monétaire qu'elle représente. La gratuité « perturbe » l'équilibre « entre ce qui est donné et ce qui est reçu » et l'individu le rétablit psychologiquement en considérant que les prestations sont moindres. 
Il faut noter que ces représentations négatives de la gratuité associée à un risque de dévalorisation de l'offre par la gratuité peuvent s'atténuer chez certains publics lors de l'expérience d'une visite gratuite, quand chez d'autres elle la renforce au contraire.

\section{La gratuité : une valorisation de l'offre}

Pour certains visiteurs ou non-visiteurs, la gratuité de l'entrée des musées apparaît d'emblée "intéressante». Elle est supposée inciter à fréquenter les musées et peut constituer " une occasion », autrement dit une opportunité, un intérêt en soi, une aubaine, voire une "bonne affaire » (Schindler, 1989).

La gratuité peut également valoriser la visite des musées en permettant de vivre une expérience à travers des sensations que le visiteur découvre spécifiquement dans ce contexte de gratuité. La gratuité permet aussi de «prendre son temps » pour visiter un musée. Elle permet ainsi de passer moins de temps dans une visite (" on ne se sent pas obligé d'aller jusqu'au bout »). Éventuellement, il est possible de revenir plusieurs fois. Ainsi, la gratuité modifie-t-elle la façon dont le visiteur perçoit l'usage des lieux. De plus, la gratuité encourage le lien social. Elle favorise, par exemple, la visite en famille ou en groupe.

En supprimant l'échange contraint d'argent, la gratuité représente alors un élément clé de contribution à l'expérience partagée et, plus spécifiquement, au lien social. Nous retrouvons ainsi différentes composantes de la valeur identifiées par différents auteurs (Aurier, Evrard et N'Goala, 2004 ; Bourgeon, 2005), notamment la valeur utilitaire, la connaissance, la stimulation expérientielle, la valeur de lien social, d'expression de soi et la spiritualité.

Nous constatons donc à nouveau une évaluation globale du compromis entre les bénéfices et les efforts ou sacrifices perçus. D’une part, la suppression du prix d'entrée augmente directement la valeur perçue, indépendamment de ses autres composantes. La valeur perçue est associée au prix monétaire le plus bas possible : "c'est gratuit, c'est intéressant, on y va». D'autre part, la suppression du prix d'entrée peut être mise en perspective avec ce qui est susceptible d'être offert par le musée : prestige des lieux, notoriété, originalité des œuvres, accueil, information, services... La valeur perçue est alors associée à la qualité obtenue pour le prix monétaire payé. La gratuité n'est pas supposée dégrader ce qui est offert : elle augmente donc la valeur perçue. Enfin, il faut remarquer que la gratuité peut être perçue par certains publics comme un attribut, voire une valeur en soi. Elle peut alors augmenter la valeur perçue car les individus la considèrent alors comme un attribut de l'offre qui domine les autres.

\section{La gratuité : un impact neutre sur la valeur de l'offre}

Pour certains publics, qu'ils soient pour ou contre la gratuité à l'entrée, la valeur des musées et de l'expérience de visite est déconnectée du prix à payer. Les discours de certains visiteurs mettent ainsi en évidence que la valeur attachée à l'objet de consommation dépasse le seul cadre du prix ou du fait de payer. L'absence de sacrifice monétaire, que représente la gratuité, est alors indépendante de la valeur attachée aux musées. Comme disent ces visiteurs, « ce ne sera pas du tout un critère d'envie, de motivation, dans ma façon de découvrir ces lieux-là »; "la gratuité n'est pas la motivation pour laquelle on vient ou on ne vient pas au musée "; "ça ne changera pas mon comportement par rapport à la visite ».

La valeur peut être reliée plus spécifiquement à l'orientation intrinsèque de la motivation. La visite d'un musée est alors « consommée » ou vécue comme une fin en soi, sans but utilitaire.

Par conséquent, ce lien entre les représentations de la gratuité et la valeur attachée aux musées et à l'expérience de visite peut être compris par le glissement de la relation duale «valeur d'usage/valeur d'échange » vers celle de «valeur signe/valeur d'échange symbolique » correspondant à la relation « signifiant/signifié » de l'objet de consommation (Baudrillard, 1972). Il est ainsi possible que la demande des individus soit insensible au prix d'un billet d'entrée dans un musée et davantage sensible à la valeur d'échange symbolique.

\section{Conclusion}

À partir des développements précédents, nous constatons d'abord que la valeur des musées et de leur expérience de visite est perçue par les publics selon un axe principal : la reconnaissance de la valeur de l'offre des musées d'un point de vue global, dans la perspective d'une relation d'échange, entre les musées et leurs publics et, plus spécifiquement, dans une approche expérientielle, lors du vécu de la visite. La reconnaissance des musées par leurs publics, visiteurs ou nonvisiteurs, comme une valeur collective symbolique d'un patrimoine ou d'une culture est présente dans les représentations, mais secondaire. Cette première 


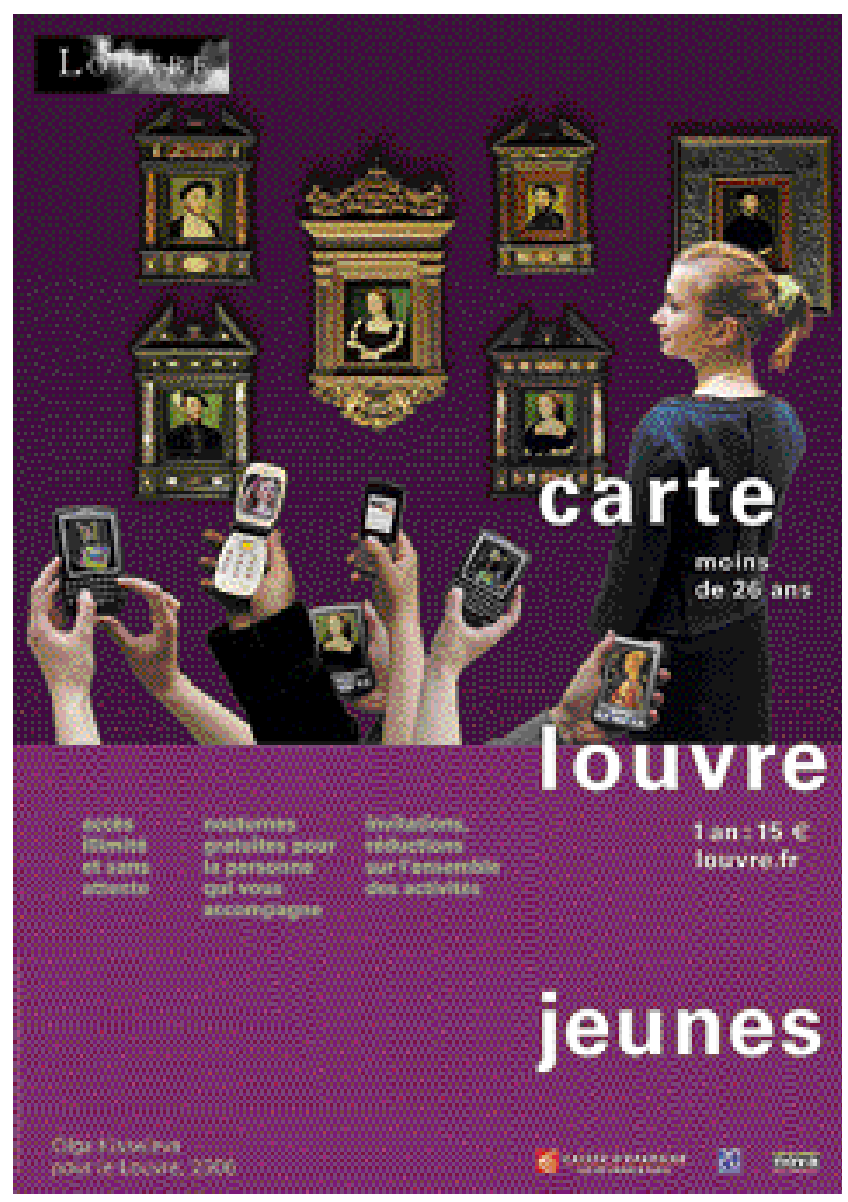

contribution de la recherche sur le lien gratuité des musées-valeur perçue par les publics relativise donc l'importance traditionnelle accordée par les professionnels des musées à la seule «valeur d'existence » de ces musées (Greffe, 2003), ciment d'une identité collective, et témoigne du fait que la société contemporaine les inscrit aussi dans une valeur d'usage et d'échange, et que cela constitue un élément important du changement de leur identité. Combattre cette évolution revient à ignorer les représentations des publics. Lintégrer permet au contraire de mieux communiquer auprès d'eux la valeur d'existence des musées.

Nos analyses permettent ensuite de montrer comment, dans les représentations des publics, la gratuité est fortement associée à la valeur des musées : - pour certains, la gratuité peut être perçue comme un risque de dévalorisation des musées : au plan symbolique, elle modifie le statut du lieu, en le désacralisant et en banalisant la visite ; au plan matériel, elle est perçue comme un facteur d'explication d'un niveau de services moindre et elle transforme les conditions de l'expérience, en pourvoyant des éléments perçus comme gênants (affluence, publics différents, contraintes de planification...).
- pour d'autres, la gratuité est un facteur de valorisation des musées : elle contribue à en faire des espaces non marchands, des lieux à part et à susciter, chez les publics, des expériences partagées. La gratuité apporte une valeur de convivialité : elle crée du lien social ;

- enfin pour d'autres encore, la gratuité peut n'avoir aucun effet : le prix à l'entrée n'influence pas la perception de la valeur des musées et des monuments et de leur visite. Pour eux, peu importe qu'il y ait gratuité ou non, leur comportement de fréquentation ne changera pas.

Signalons, que cette deuxième contribution de la recherche a été approfondie dans l'ensemble de la recherche (Gombault, Petr, Bourgeon-Renault, Le Gall et Urbain, 2006) : ces représentations de la gratuité recueillies hors site ont été interrogées et comparées avec les représentations et l'expérience vécue pendant la visite gratuite d'un musée. Les analyses, qui ne sont pas développées ici, montrent que l'expérience de la visite gratuite peut modifier les représentations des musées et leur projet d'usage chez certains visiteurs, de différentes façons.

Plus largement, l'impact des représentations de la gratuité à l'entrée des musées sur la perception de la valeur de l'offre par les publics demanderait à être davantage précisée par une enquête quantitative descriptive complémentaire visant à identifier le profil des publics selon leurs représentations. Celle que nous avons réalisée dans l'étude, croisée avec les protocoles qualitatifs, permet d'amener des éléments, notamment par l'identification d'univers de représentations des musées : par exemple nous savons que ce sont plutôt des visiteurs habitués, appartenant à l'univers sacré, qui déclarent que la gratuité valorise les musées et leur expérience de visite, que c'est majoritairement pour les non-visiteurs, qu'elle a un impact neutre... Cependant, les profils sont plus contrastés pour les visiteurs qui pensent qu'elle dévalorise l'offre : visiteurs habitués et occasionnels issus des univers patrimonial et loisirs, se mêlant dans cette représentation commune.

Ces tendances demandent à être affinées et approfondies sur plusieurs points : quelles sont les différences de représentations du lien gratuité des musées-valeur perçue par les publics liées à la zone géographique et à la densité muséale de la zone ? Ces représentations varient-elles dans un contexte touristique ou non ? Y a-t-il plus de craintes de valorisation/dévalorisation quand on parle pour soi ou pour 
les autres (les résultats de la recherche montrant ce double niveau de discours dans les représentations en général) ? Une analyse quantitative des représentations des publics de ce lien représentations de la gratuité des musées-valeur perçue permettrait aux établissements d'envisager une politique de gratuité plus ciblée et de réfléchir à des réponses adaptées pour optimiser ses effets de valorisation des musées et de l'expérience de visite et pour limiter ses effets de dévalorisation.

\section{Note}

(1) L'italique signale des verbatim, extraits des entretiens individuels ou de groupes, réalisés hors site.

\section{Bibliographie}

Aurier, P., Évrard, Y. et N'Goala, G. Comprendre et mesurer la valeur du point de vue du consommateur, Recherche et Applications en Marketing, $\mathrm{n}^{\circ} 19,2004$, pp. 1-20.

Bailey, S., Falconer, P., Foley, M., McPherson, G. et Graham, M. Charging for admission to museums and galleries : arguments and evidence, Museum Management and Curatorship, $\mathrm{n}^{\circ} 16$, 1997, pp. 355-369.

Baudrillard, J. Pour une critique de l'économie politique du signe. Paris : Gallimard, Les Essais, 1972.

Bourgeon, D. Du marketing expérientiel appliqué aux musées, Revue Espaces, novembre 2005, pp. 41-47.

Dickenson, V. The Economics of museum admission charges, Curator, n³6/3, 1993, pp. 220-234.

Godbout, J.-T. L'esprit du don. Paris : La Découverte Poche, 2000.
Gombault, A. La gratuité dans les musées : une revue internationale, Les institutions culturelles au plus près du public, Fourteau, C. (dir.), La Documentation française, 2002.

Gombault, A. La gratuité au cœur de la stratégie de prix du musée, Revue Espaces, 2005, pp. 38-43.

Gombault ,A., Petr, C., Bourgeon-Renault, D., Le Gall, M. et Urbain, C. La gratuité des musées et des monuments côté publics Représentations, projets d'usage et comportements des publics, Octobre S. et Rouet, F. (dir.), département des Études, de la Prospective et des Statistiques, ministère de la Culture et de la Communication, Paris : La Documentation française, collection Questions de Culture, 2006.

Gottesdiener, H. et Godrèche, N. Les dimanches gratuits du musée du Louvre, enquête qualitative. Paris : musée du Louvre, service culturel, CEREM, 1996.

Greffe, X. La valorisation économique du patrimoine. Paris : La Documentation française, 2003.

Holbrook, M.-B. et Hirschman, E.-C. The Experiential Aspects of Consumption : Consumer Fantasies, Feelings and Fun, Journal of Consumer Research, n9, 1982, pp. 132-140.

Homans, G.-C. Social Behavior, Its Elementary Forms. London : Routledge \& Kegan Paul London, 1961.

Schindler, R.-M. The excitement of getting a bargain : some hypotheses concerning the origins and effects of smart-shopper feelings, Advances in Consumer Research, nº16, 1989, pp. 447-451.

Sheth, J.-N., Newman, B.-I. et Gross, B.-L. Why we buy what we buy : a theory of consumption values, Journal of Business Research, n²2, 1991, pp. 159-170.

Zeithaml, V.-A. Consumer perceptions of price, quality, and value : a means-end model and synthesis of evidence, Journal of Marketing, n52, 1988, pp. 2-22. 